Results 199 substitution proposals were sent to the physicians (51.8\% accepted, $48.2 \%$ not accepted. Of these, in $17.1 \%$ of cases the patient brought the medicine from home and in $7 \%$ treatment was discontinued).

The most common clinical justification accepted (8 cases) was leg oedema caused by amlodipine (maintenance of manidipine). The second one was anaerobic infection where levofloxacin is not active (maintenance of moxifloxacin).

The global DNI price within two months of study was $€ 1,148.78$. The cost saving with the acceptance of $51.8 \%$ of substitutions was $€ 472.63$ in two months. If $100 \%$ of substitutions had been accepted, the therapeutic equivalent prescription would have saved $€ 586.75$.

In $17 \%$ of cases therapeutic equivalents were prescribed at discharge.

Conclusions The suggested substitution was accepted in more than half of cases.

The adjustment of medical prescriptions to the hospital's pharmacotherapeutic guide prevailed over the economic saving, which was not significant.

The prescription of therapeutic equivalents at discharge was not as expected.

No conflict of interest.

\section{OHP-015 CLINICAL RESEARCH IN FRANCE AND QUEBEC}

doi:10.1136/ejhpharm-2013-000276.389

'A Guérin, ' $\mathrm{C}$ Tanguay, 'D Lebel, ${ }^{2} \mathrm{O}$ Bourdon, ${ }^{1 J F}$ Bussières. ${ }^{1} \mathrm{CHU}$ Sainte-Justine, Pharmacy, Montreal, Canada; ${ }^{2}$ Hôpital Universitaire Robert-Debré, Pharmacy, Paris, France

Background Pharmacy practise is evolving in most countries. Hospital pharmacists are pivotal in the organisation and the support of clinical trials. We looked at the current state of pharmacy practise in clinical research

Purpose To identify differences in clinical research organisation and pharmacy practise between France and Quebec (Canada).

Materials and Methods This is a descriptive study. A literature review was performed in order to describe the organisation of clinical research and the role of pharmacists in clinical research for both countries. Differences were identified by a panel consisting of one French pharmacy intern, one French hospital pharmacist, one Quebec research assistant and two Quebec hospital pharmacists.

Results Fourteen differences relating to research organisation were identified. France and Canada have different normative frameworks, regulatory authorities, authorization processes, delays and shutdown processes. While it is encouraged, clinical trial registration is not mandatory in Canada. Data needs to be archived for 15 years in France vs. 25 years in Canada. Institutional review boards (IRB) have different names, location, composition, nomination processes, mandate duration and informed consent processes for minors. Seven key differences in pharmacy practise were identified. There are different authorization processes for drug compounding and manufacturing. Pharmacy fees are based on a national reference in France, but not in Canada. Software for the computerization of pharmacy services for clinical trials is common in France. In addition to drug trials, French pharmacists also manage sterile medical devices and medicinal products derived from human blood. Canadian pharmacists offer decentralised pharmaceutical care to hospitalised patients. Canadian pharmacists can be principal investigators if a doctor is the qualified investigator.

Conclusions Clinical research organisation is similar on many aspects, but 21 main differences were identified. Comparisons between countries help identify best practise and may contribute to practise improvement.

No conflict of interest.

\section{OHP-016 CONSUMPTION OF OPIOID ANALGESICS IN HOSPITAL PHARMACY AND CONSULTATIVE CARE FOR PATIENTS}

doi:10.1136/ejhpharm-2013-000276.390

1J Sumberova, ${ }^{2} \mathrm{M}$ Lzicar, ${ }^{3} \mathrm{~J}$ Kolar. 'Pharmacy Konstancie, Community Pharmacy, Brno, Czech Republic; '2St. Ann's University Hospital, Hospital Pharmacy, Brno, Czech Republic; ${ }^{3}$ University of Veterinary and Pharmaceutical Sciences, Faculty of Pharmacy, Brno, Czech Republic

Background In recent years the incidence of different types of pain is increasing. We have found the same in the St. Anne's hospital in the Czech Republic. Patients are now able to ask about the correct usage of opioid drugs in pharmacy consultation centre, which opened in 2011.

Purpose To find out the consumption of opioid analgesics from 2008 to 2011. This is an analysis of prescriptions by doctors from the pain treatment centre. We also collected data from patient records in the pharmacy consultation centre and we wanted to know how many patients come to consult us.

Materials and Methods Data were obtained from the pharmacy computer software. We made a retrospective evaluation, calculated the defined daily dosage (DDD) and compared consumption of opioid analgesics during 2008-2011 for ATC class N02A and other subclasses. We analysed the consultation records.

Results Consumption of weak opioids decreased over that time, while consumption of strong opioids increased, which had to be prescribed. Opiates were prescribed more often to women. The highest consumption was of buprenorphine, than fentanyl and oxycodone, from weak opioids it was tramadol. Consumption of fentanyl increased from 35735 DDD (2010) to 39924 DDD (2011), while buprenorphine consumption decreased from 45059 DDD (2010) to 38675 DDD (2011). The amount of morphine used last year was twice that of previous years. The total number of patients who visited the pharmacy consulting centre was 41 , six patients were from the pain treatment centre. Average age was 61.3. Patients wanted to control interactions, secondly combat adverse effects of opioids and requested information about neuropathic pain. Average consultation length was 22.5 minutes.

Conclusions The consumption of strong opioids is gradually increasing, doctors follow guidelines and they aren't afraid of prescribing strong opioids. In future it would be appropriate to extend the distribution of informatory materials by the consultation centre - not only about the opioid analgesics.

No conflict of interest.

\section{OHP-017 COST COMPARISON OF INTRAVITREAL ANTIANGIOGENIC DRUGS IN THE TREATMENT OF AGE-RELATED MACULAR DEGENERATION}

doi:10.1136/ejhpharm-2013-000276.391

'C Matallana, 'MC Izquierdo, 'V Martinez, 'M Hernando, ${ }^{2 P}$ Aznarte, ${ }^{3} \mathrm{MT}$ Sanchez. ${ }^{1}$ Hospital Clínico Universitario, Pharmacy, Valladolid, Spain; ${ }^{2}$ Hospital Virgen de las Nieves, Pharmacy, Granada, Spain; ${ }^{3}$ Hospital Clinico Universitario, Pharmacy, Valladolid, Spain

Background The primary treatment of Age-related Macular Degeneration (AMD) is based on inhibition of Vascular Epithelial Growth Factor (VEGF) with antiangiogenic drugs, which delay disease progression and improve the patient's vision.

Choosing between bevacizumab and ranibizumab is still up for debate. Bevacizumab has not been approved for $\mathrm{AMD}$, while ranibizumab has a safer profile and is legally approved for this condition, although it is more expensive.

Purpose To evaluate the cost of intravitreal ranibizumab in AMD and to compare with the hypothetical cost of treatment with intravitreal bevacizumab in off-label conditions for the same group of patients. 